\title{
GPPS-BJ-2019-0008
}

\section{SHOCK-VORTEX INTERACTION IN A TRANSONIC TURBINE CASCADE}

\author{
Dajan Mimic, Marcel Oettinger, Philipp Sauer, Florian Herbst \\ Junior Research Group Multiphysics of Turbulent Flows \\ Institute of Turbomachinery and Fluid Dynamics \\ Leibniz Universität Hannover \\ mimic@tfd.uni-hannover.de \\ Hanover, 30167, Germany
}

\begin{abstract}
In this paper, we have investigated numerically the interaction between secondary flow and a normal compression shock in a transonic turbine cascade.

We observe that the sudden deceleration caused by the compression shock triggers an instability in weaker regions of the vortex system. This instability forms a pattern of concentric rings of elevated vorticity, which, in turn, cause the shock to deform into the same concentric wave-like pattern. The same pattern appears in the entropy distribution just downstream of the shock as a result of the uneven shock-intensity distribution.
\end{abstract}

\section{INTRODUCTION}

The trend towards increasingly high circumferential velocities in turbines yields ever-increasing Mach numbers and transonic flow. The interaction of compression shocks and secondary-flow vortices poses a great challenge to aerodynamicists, turbulence modellers and turbine designers, alike. However, to derive more accurate turbulence models to predict the development of turbulence - and, in extension, secondary flowacross compression shocks it is necessary to acquire a more thorough understanding of the non-linear flow phenomena causing instabilities at the shock front.

While the mechanisms involved in the interaction between vortices and compression shocks are generally welldocumented, they seldom undergo detailed discussion in the context of turbine aerodynamics.

In the realm of compressors, in turn, this topic moved into the focus of research in the late 1990s. Puterbaugh and Brendel (1997) explain in an impressive manner how the tip leakage vortex in a compressor cascade leads to a bulging of the shock front-a phenomenon which is apparently not related to the tangential velocity distribution of the vortex, but entirely to the axial-velocity deficit that the vortex core carries. The authors further suggest that the interaction is of entirely inviscid nature, as it can be explained solely by the abrupt increase in pressure encountered by the vortex.

Highly resolved simulations of the interaction between a slender, longitudinal vortex and a normal shock by Thomer et al. (2001) partially confirm these findings: as the vortex impinges upon the shock, the shock bulges upstream. In the case of a vortex breakdown, a recirculation zone forms directly downstream of the shock, causing the shock to curve even further upstream.

The computations were performed using Navier-Stokes and Euler equations with very similar results. This similarity suggests that viscous effects do indeed play a subordinate role. The authors also confirm the positive correlation between the axial-momentum deficit of the vortex and vortex breakdown. In contrast to Puterbaugh and Brendel (1997), however, the authors did find a link between the circulation-the tangential velocity, by implication - and vortex breakdown: higher circulations favour the breakdown of the vortex. The general topology of interactions between longitudinal vortices and normal shocks was later confirmed by Zudov (2011) using direct numerical simulation (DNS).

\section{Transonic turbines}

Transonic flow plays a great role in high-pressure turbine aerodynamics - and with the development of geared turbofans increasingly so in low-pressure turbines-which certainly is reflected in the literature. Graham and Kost (1979) showed, using Schlieren photographs and pneumatic mid-span measurements, that shock-wave-boundary-layer interactions can act as a considerable loss-generating mechanism in transonic turbine 
blading. The authors stressed the necessity to better understand three-dimensional flow phenomena in transonic turbine flow.

Subsequently, Perdichizzi (1989) investigated the impact of a Mach-number variation on the secondary flow measured downstream of a turbine cascade. The results of this study convey that the passage vortices wander closer to the end-walls as the Mach number increases. At the same time, trailingshed and corner vortices gain a more dominant role in the secondary flow system. The author suggests that the shockwave-boundary-layer interaction introduces another important source of three-dimensionality into the flow.

Arts et al. (1990) presented a comprehensive series of mid-span measurements for the transonic VKI LS89 aerofoil. Due to the high quality of the experiments and the excellent documentation, these cascade measurements often serve as the validation and basis for further numerical studies. For the same reasons, the investigations in this paper are based on this profile.

Detemple-Laake (1991) put forward a detailed analysis of the flow in a transonic turbine cascade using a wide array of experimental techniques, including surface oil flow patterns, end-wall pressure taps and span-wise traverses to gain an understanding of the secondary flow structures in such a cascade. A comparison to subsonic operation of the cascade showed that an increase of the Mach number leads to a shift of the passage vortices towards the end-walls until the passage chokes. Endwall measurements exhibited an impact from the trailing shock system - the so-called fish-tail shocks. Conversely, Moustapha et al. (1993) found no impact of the exit Mach number on the secondary flow structures in a low-aspect-ratio turbine cascade.

\section{APPROACH}

Secondary-flow vortices and turbulence form a close bond with each other, as the time-averaged properties of turbulent flow dictates the behaviour of the large vortical structures by impacting their diffusion and dissipation. Likewise, smallscale turbulent structures feed on the kinetic energy of largescale vortices. In this study, Reynolds-averaged Navier-Stokes (RANS) simulations serve as an exploratory tool to gain an impression of the large-scale processes.

Secondary flow, which forms as the incoming end-wall boundary layers get twisted and skewed by the turning in the passage, and its interaction with a compression shock occuring in the passage shall lie in the focus of this paper. A thorough understanding of the mechanisms is invaluable for improvement of three-dimensional blade designs in high-pressure turbine and transonic low-pressure turbines.

When trying to describe the shock-wave-vortex interaction, two questions can be posed:

1. How do the vortices impact the shock?

2. How does the shock influence the vortices?

To answer these questions, it is necessary to isolate the impacting factors as far as possible. In this paper, we chose to simulate the interaction between secondary-flow vortices and a compression shock in a transonic turbine cascade-and to compare this realistic flow field to a simplified model without any secondary flow. This gives us the opportunity to observe the mostly undisturbed compression shock and to subsequently carve out the effects that are caused by the shock-vortex interaction.

The transonic VKI LS89 cascade (Arts et al., 1990) was used as the test case for the investigations presented in this paper, since it is splendidly documented. The cascade was simulated as a fully three-dimensional case with a channel height of $h=100 \mathrm{~mm}$ and a chord length of $\ell=67.647 \mathrm{~mm}$. With a stagger angle of $55 \mathrm{deg}$, this results in an axial chord length of $36.985 \mathrm{~mm}$.

Specifically, we chose to simulate the transonic test case MUR047 with an isentropic outlet Mach number

$$
\mathrm{Ma}_{\mathrm{is}} \equiv \sqrt{\frac{2}{\kappa-1}\left(\frac{p_{\text {tot,in }}}{p}\right)^{\frac{\kappa-1}{\kappa}}-1}
$$

of $\mathrm{Ma}_{\text {is,out }}=1.02$. The test case was simulated without inlet boundary layers and with inviscid end walls in order to suppress the formation of secondary flow and obtain an idealised reference case. As the next step, we included boundary layers in the inlet boundary conditions and treated the end walls as viscous to generate a realistic passage flow field; this numerical test case is referred to as MUR047BL (see Tab. 1).

\section{Table 1 Test cases investigated}

\begin{tabular}{ccc}
\hline Case name & End-wall boundary layers & Secondary flow \\
\hline MUR047 & no & no \\
MUR047BL & yes & yes \\
\hline
\end{tabular}

\section{Computations}

The fully compressible, finite volume solver TRACE 9.1.538 - a development of the German Aerospace Center (DLR) — was used for all simulations. Turbulence was modeled with the $2003 k-\omega$ shear stress transport (SST) turbulence model by Menter (1994) in the version presented by Menter et al. (2003) without any special transition modelling. Realisability in the stagnation point region was achieved by using the stagnation point anomaly fix by Kato and Launder (1993).

A second-order accuracy implicit finite volume spatial discretisation was used together with a third-order differencing scheme and a van Albada square limiter to compute the state variables. The incomplete lower-upper (ILU) factorisation method was chosen to solve the governing equations. 


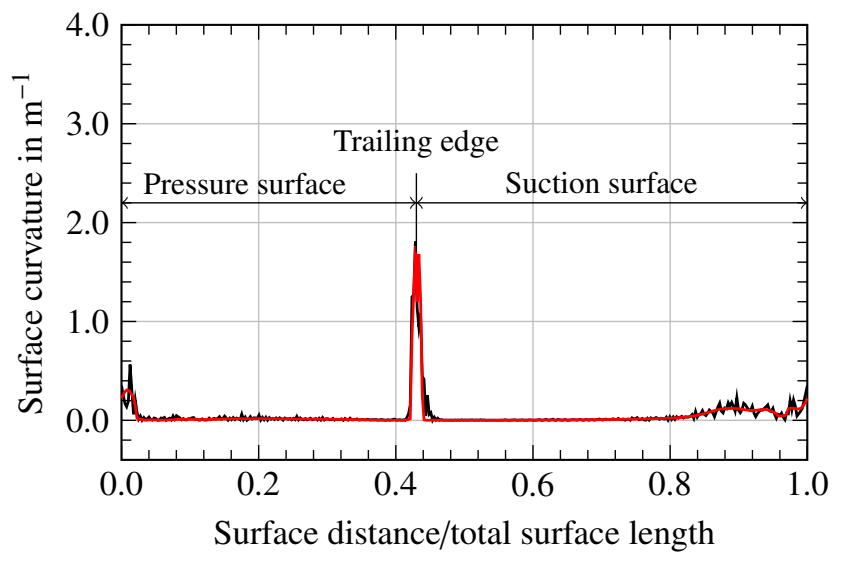

- Published coordinates plotted using $3^{\text {rd }}$ order spline Interpolated coordinates plotted using $3^{\text {rd }}$ order spline

\section{Figure 1 Surface curvature distribution of published coordinates for VKI LS89 aerofoil and modified coordinates using a $3^{\text {rd }}$ order spline}

As Wheeler et al. (2016) pointed out, the manufacturing coordinates published by Arts et al. (1990) exhibit high oscillations. For this reason, we performed a similar geometry optimisation as Wheeler et al. (2016) with similar results. A comparison between the geometry variants is shown in Fig. 1.

The mesh used for the studies in this paper comprises approximately $3.5 \times 10^{6}$ cells. The simulations converged to maximum residuals of $L_{\infty}<9 \times 10^{-14}$ and mean residuals of $L_{1}<4 \times 10^{-16}$.

\section{Grid-convergence study}

The grid-convergence index (GCI) according to Roache (1994) has been calculated for the pitch-wise blade force, i.e., the lift $F_{y}$ and mass-flow rate $\dot{m}$ to assess the influence of spatial discretization. The grid study was conducted for MUR047BL.

Table 2 Grid-convergence indices of characteristic parameters

\begin{tabular}{rcl}
\hline & $F_{y}$ & \multicolumn{1}{c}{$\dot{m}$} \\
\hline$e_{a}^{21}$ & 166.55 & 0.4628 \\
$e_{a}^{32}$ & 166.39 & 0.4629 \\
$\varrho$ & 0.93 & 3.09 \\
$\mathrm{EERE}_{21}$ & 0.0009 & 0.0002 \\
$\mathrm{EERE}_{32}$ & 0.0012 & 0.0001 \\
$\mathrm{GCI}_{21}$ & -0.0034 & -0.0044 \\
$\mathrm{GCI}_{32}$ & -0.0008 & -0.0008 \\
\hline
\end{tabular}

The lift is proportional to the intensity of secondary flow, which is of primary concern for the present variation, while the mass flow rate is a more basic characteristic variable. The GCI is defined as

$$
\mathrm{GCI} \equiv \frac{\mathfrak{F}_{\mathrm{S}} e_{\mathrm{a}}}{\Re \varrho-1}
$$

Three grids of different spatial resolution are used for the grid study with the total node count being $N_{1}<N_{2}<N_{3}$ as detailed in Tab. 2. The refinement factors are equal to $\Re_{32}=2 / 3$ and $\Re_{12}=0.5$. In accordance with the recommendations of the ASME V\&V Committee (2009), a factor of safety $\mathfrak{\mho}_{\mathrm{s}}=1.25$ is used to calculate the GCI. For both characteristic parameters, the relative error $e_{a}$ and, thus, the discretization error decrease with grid refinement. The absolute differences are minor as expected. For the medium grid resolution $N_{2}$, the error relative to the estimated Richardson result is $\mathrm{EERE}_{21}=0.09 \%$ for the lift force and $\mathrm{EERE}_{21}=0.02 \%$ for the mass-flow rate. While the finest grid shows a lower relative error, the difference is minor and does not justify the considerable increase in computational resources required. Therefore, the medium grid is used for all of the following computational studies.

\section{Boundary conditions}

Arts et al. (1990) provide only mid-span values for the inlet boundary conditions of the VKI LS89 test cases. These values were used over the full channel height for the test case MUR047. All boundary conditions, including the outlet static pressure, of MUR047 are given in Tab. 3.

Table 3 Boundary conditions for the MUR047 test case

\begin{tabular}{lcl}
\hline Parameter & Symbol & Value \\
\hline Inlet total temperature & $T_{\text {tot,in }}$ & $420 \mathrm{~K}$ \\
Inlet total pressure & $p_{\text {tot,in }}$ & $1.596 \times 10^{5} \mathrm{~Pa}$ \\
Inlet pitch-wise flow angle & $\alpha_{\text {in }}$ & $0 \mathrm{deg}$ \\
Inlet turbulence intensity & $\mathrm{Tu}$ & $3 \%$ \\
Outlet static pressure & $p_{\text {out }}$ & $8.2357 \times 10^{4} \mathrm{~Pa}$ \\
\hline
\end{tabular}

However, as secondary flow intensity strongly depends on the state and thickness of inlet boundary layers, a precursor simulation was used to generate the radial inflow profiles for MUR047BL. Based on technical drawings in the original publications, we inferred an intake length of approximately $1 \mathrm{~m}$ upstream of the inlet plane. Total pressure, total temperature, turbulent kinetic energy and turbulent length scale at the inlet of the precursor simulation were iteratively adapted until the desired mid-span values were obtained at the outlet of the domain. A turbulent length scale of $l_{\text {turb }}=3.221928 \times 10^{-3} \mathrm{~m}$ at 


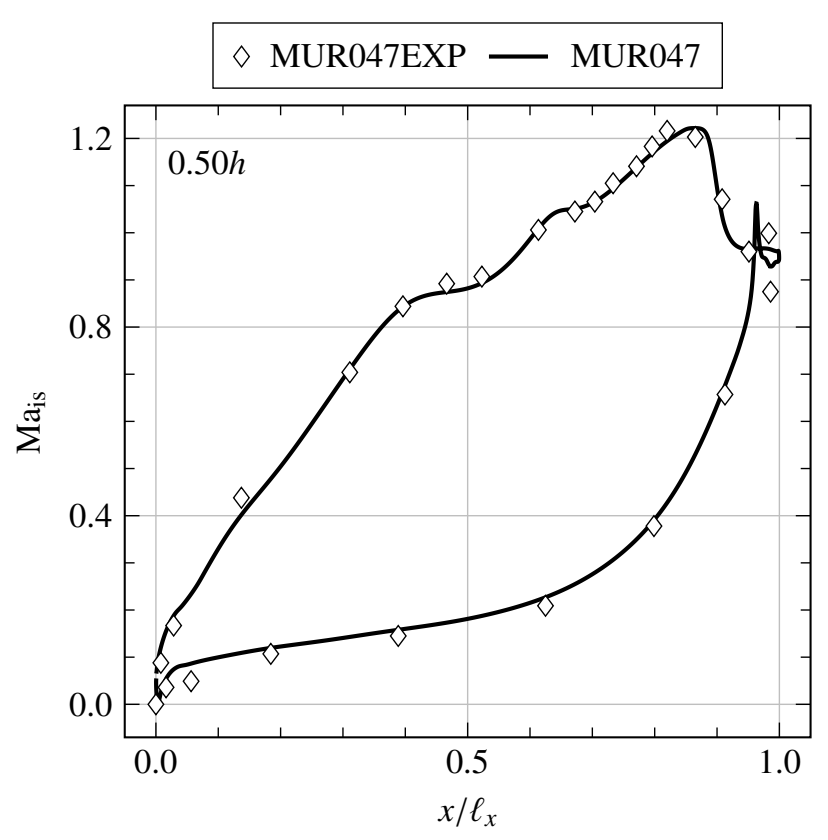

Figure 2 Validation of isentropic Mach number distribution at mid-span

mid-span was obtained from the precursor simulation. This agrees reasonably well with values typically used. A sensitivity study, which is not shown in this paper, indicated no noticeable influence of the turbulent length scale on the isentropic Mach number distribution at mid-span. The mid-span value of the turbulent length scale was, therefore, prescribed as the inlet value for MUR047. The outlet static pressure of MUR047BL was chosen to be identical to MUR047 as an integral value over the full channel height.

\section{Validation}

Figure 2 shows the distribution of the isentropic Mach number obtained from a computation of MUR047 in comparison to the distribution measured by Arts et al. (1990) at mid-span. Evidently, the simulation captures all relevant features of the isentropic Mach number distribution with some minor discrepancies in the prediction of the shock position and intensity. The error bars of the experiment are smaller than the symbol size and, therefore, not visible.

\section{ANALYSIS}

Before focusing on a detailed discussion of the shockvortex interaction, we present an overall analysis of the impact of secondary flow on blade loading and loss generation.

\section{Profile distributions}

As can be seen from Fig. 3, the isentropic Mach number distributions remain constant along the channel height for test case MUR047. This is not surprising, as there are no spanwise inhomogeneities in the inlet flow and, thus, no secondary flow develops. The compression shock is clearly visible at

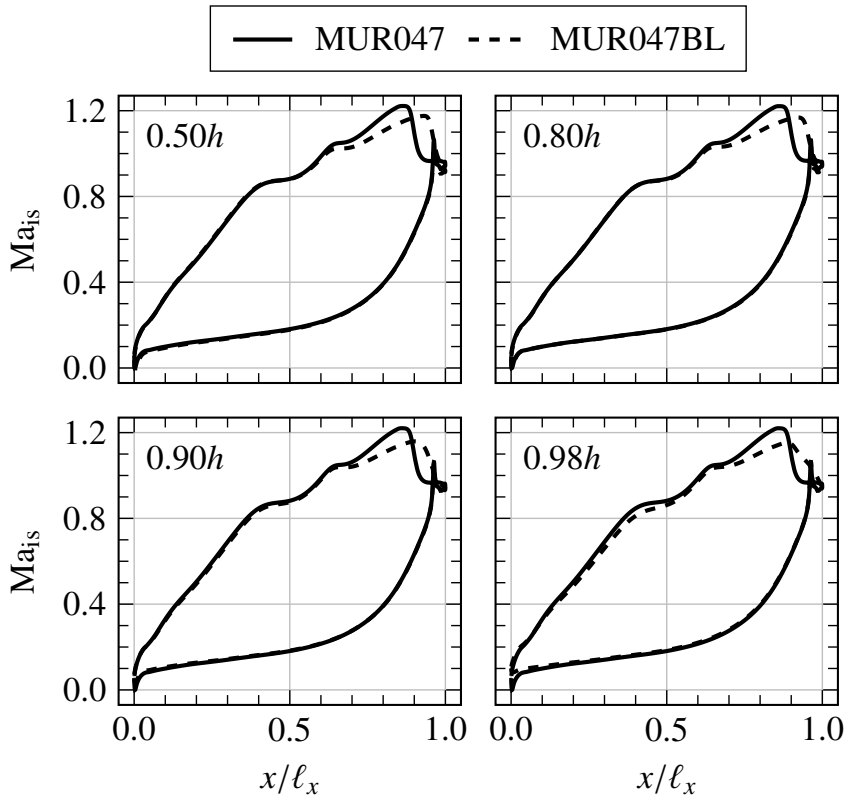

Figure 3 Isentropic Mach number distribution at different channel heights

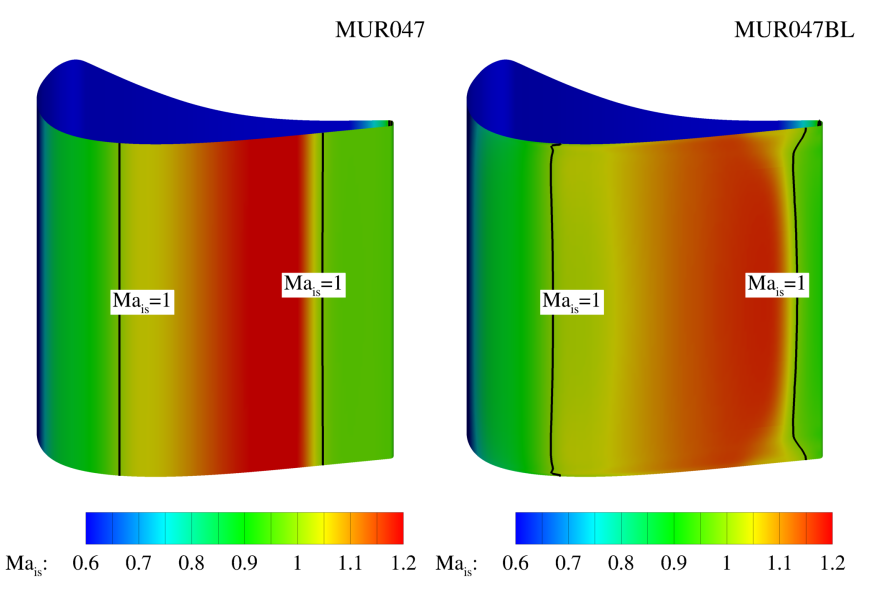

\section{Figure 4 Isentropic Mach number distribution on suction side of VKI LS89 aerofoil}

approximately $0.92 \ell_{x}$ and $0.50 h$. The peak isentropic Mach number is lower and appears later for MUR047BL; the shock position is further downstream. The discrepancies between both variants increase slightly as the extraction planes approach the full channel height. In the case of MUR047BL a slight and continuous aerodynamic unloading can be observed at around $0.4 \ell_{x}$. This is accompanied by a weakening of the compression shock, as can be seen in Fig. 4. The ensuing $\lambda$ shape near hub and tip of MUR047BL indicates the thickening of the endwall boundary layer caused by the shock-wave-boundary-layer interaction. 

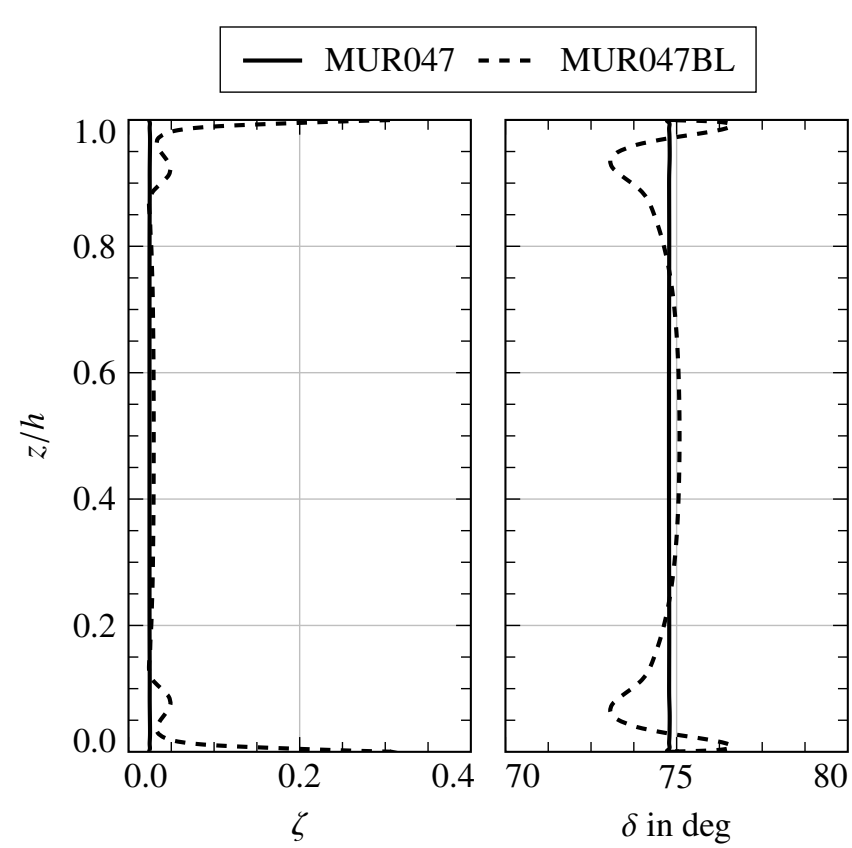

Figure 5 Radial distribution of total pressure loss coefficient (left) and turning angle (right)

\section{Radial distributions}

Figure 5 shows the mass-flow-weighted, pitch-wiseaveraged span-wise distributions of the total pressure loss coefficient

$$
\zeta \equiv \frac{p_{\text {tot,in }}-p_{\text {tot,out }}}{p_{\text {tot,in }}}
$$

and turning angle

$$
\delta \equiv \alpha_{\text {out }}-\alpha_{\text {in }},
$$

where $\alpha$ represents the pitch-wise angle with regard to the axial direction. As expected, MUR047BL exhibits noticeably increased losses due to secondary-flow vortices near the end walls and strongly increased losses due to the end-wall boundary layers directly at the end-walls. As for the turning angle, MUR047BL exhibits slightly higher turning than MUR047 in the free-stream region. Near the end walls, the flow experiences mostly reduced turning due to the secondary flow structures, with some slightly increased turning adjacent to the wall.

\section{Loss analysis}

To better quantify the individual contributions of different loss-generating phenomena-and as a handy tool for future analyses - we propose an easily applicable and generalisable method of loss breakdown. Another possible application of this approach, however, tailored for multi-stage turbomachinery, is presented in Oettinger et al. (2019).

As a starting point, we use the specific entropy to quantify the overall losses:

$$
s=c_{p} \ln \left(\frac{T}{T_{\text {ref }}}\right)-R \ln \left(\frac{p}{p_{\text {ref }}}\right) .
$$

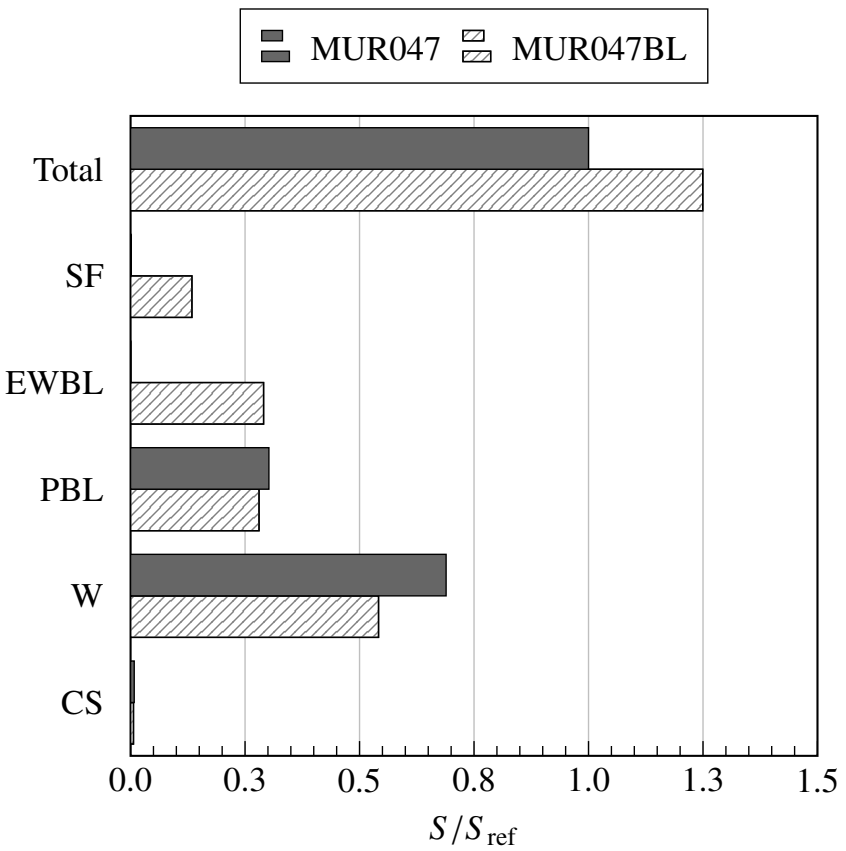

Figure 6 Loss contributions

We then define a series of detection functions in order to attribute the entropy to different sources. Note that this attribution is chosen based on phenomenological or topological considerations, not actual physical loss-generating mechanisms. The subdivisions that we use in this paper are as follows: secondary flow (SF), end-wall boundary layer (EWBL), profile boundary layer (PBL), wakes (W) and compression shocks (CS).

To subdivide the losses generated, we make use of the fact that all loss-afflicted regions (vortices, boundary layers and wakes $)$ are rotational in nature $(\boldsymbol{\Omega} \equiv \nabla \times \mathbf{c} \neq 0)$. The vorticity vector $\boldsymbol{\Omega}$ can be decomposed into a streamwise (SW) and a crosswise $(\mathrm{CW})$ component

$$
\boldsymbol{\Omega} \cdot \boldsymbol{\Omega} \equiv \Omega^{2} \equiv \Omega_{\mathrm{SW}}^{2}+\Omega_{\mathrm{CW}}^{2}
$$

The streamwise vorticity is defined as the projection of the vorticity vector onto the normalised velocity vector

$$
\Omega_{\mathrm{SW}} \equiv \frac{\boldsymbol{\Omega} \cdot \mathbf{c}}{|\mathbf{c}|}
$$

and represents a suitable measure to detect secondary-flow vortices. The crosswise component can easily be calculated per Eqn (6) and detects boundary layers and wakes. Both vorticity components are used to define a pair of weighting functions:

$$
\begin{aligned}
w_{\mathrm{SW}} & \equiv \frac{\Omega_{\mathrm{SW}}^{2}}{\max \left(\Omega^{2}, 1 \times 10^{-16}\right)}, \\
w_{\mathrm{CW}} & \equiv \frac{\Omega_{\mathrm{CW}}^{2}}{\max \left(\Omega^{2}, 1 \times 10^{-16}\right)} .
\end{aligned}
$$

The limiting function in the denominator is necessary to avoid division by zero in irrotational flow regions. 
A further flow phenomenon that requires detection is the compression shock. Here, we use a measure devised by Lovely and Haimes (1999): the Mach number immediately upstream of a compression shock is always unity perpendicular to the shock. At the same time, the local pressure gradient is always perpendicular to the shock. Therefore, the dot product of the Mach number vector and the normalised pressure gradient

$$
\sigma \equiv \frac{\mathbf{c}}{a} \cdot \frac{\nabla p}{|\nabla p|}
$$

is always unity just at the shock. This criterion is used to define a Boolean function as per Eqn (12) in Appendix A. The Boolean blending functions to determine, whether losses are counted as part of EWBL, PBL or W are given in Eqns (13)(15) in Appendix A. The different blending functions are used as weighting functions to weight the specific entropy. The overall entropy of MUR047 serves as the reference $S_{\text {ref }}$. An overview of the individual definitions is given in Eqns (16)-(20) in Appendix B. The respective specific entropies are used to calculate density-weighted spatial integrals according to

$$
S_{\square} \equiv \int_{V} \rho s_{\square} \mathrm{d} V,
$$

where $\square$ is a placeholder for the respective indices SF, EWBL, PBL, W, CS or the overall entropy.

The result of this analysis is presented in Fig. 6. As a general sanity check, the overall entropy increases for MUR047BL. It is striking that the CS losses can be neglected. This is because the Mach number just upstream of the shock approaches unity, leading to a very weak shock. The resulting entropy production across the shock is, therefore, expectedly small. While the PBL losses remain constant across all test cases, there is a reduction in $\mathrm{W}$ losses-which represent the dominant loss-generating mechanism in both cases-when secondary flow is introduced. This is most probably due to the unloading of the near-wall blade regions in the presence of secondary flow. As expected, the greatest differences between test cases arise in the direct SF losses and the EWBL losses. A depiction of the evolution of the SF losses through the passage is shown in Fig. 7. Evidently, the horse-shoe vortex and passage vortex represent the dominant entropy-generating secondary flow structures in the passage, indicating that the criteria devised for loss separation do, indeed, work as expected.

\section{Shock-vortex interaction}

While the shock-induced losses are negligible in this test case, the interaction between compression shock and secondaryflow vortices still gives interesting results. Moreover, these results could be of greater importance in other instances, where the shock-induced losses are more pronounced.

MUR047. As a starting point, we consider the reference MUR047 without inlet boundary layers and with inviscid end-

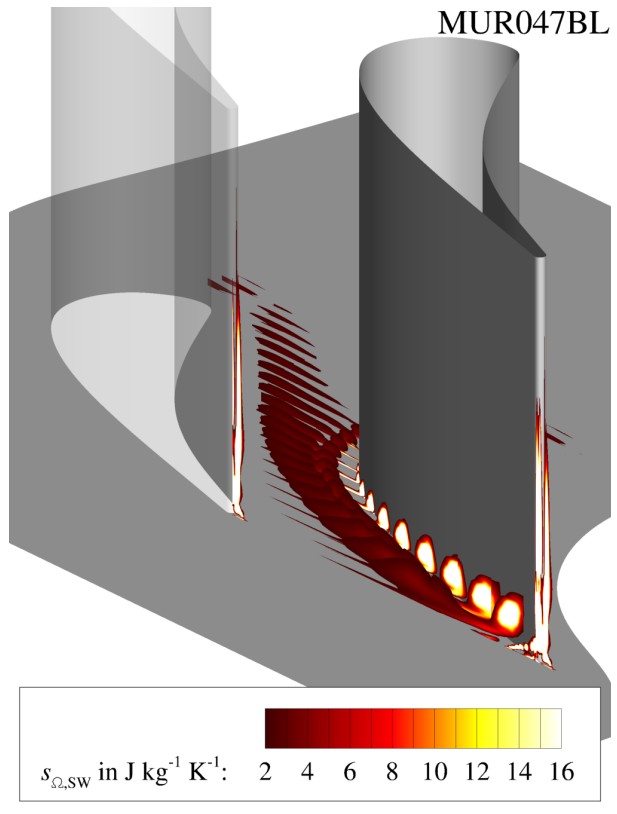

Figure 7 Specific entropy due to
secondary flow in passage

wall boundary conditions. Figure 8 shows the shock by blanking cells that have a primary cell value of $\sigma<1$ (see Eqn 10 for definition of $\sigma$ ). It is clear that the shock forms an approximately flat surface, only disrupted by the subsonic wake flow of the neighbouring blade. Also the $\lambda$-shock formation can be seen where the shock meets the profile boundary layer.

MUR047BL. In the case of MUR047BL, the shock front exhibits a span-wise curvature due to the inhomogeneous velocity distribution. There is, however, another, more surprising effect: the shock surface forms a complex structure, characterised by concentric "ripples" that seem to emanate from the center of the passage (see Fig. 8). The same structure can also be seen in the superimposed contour map denoting the distribution of specific entropy.

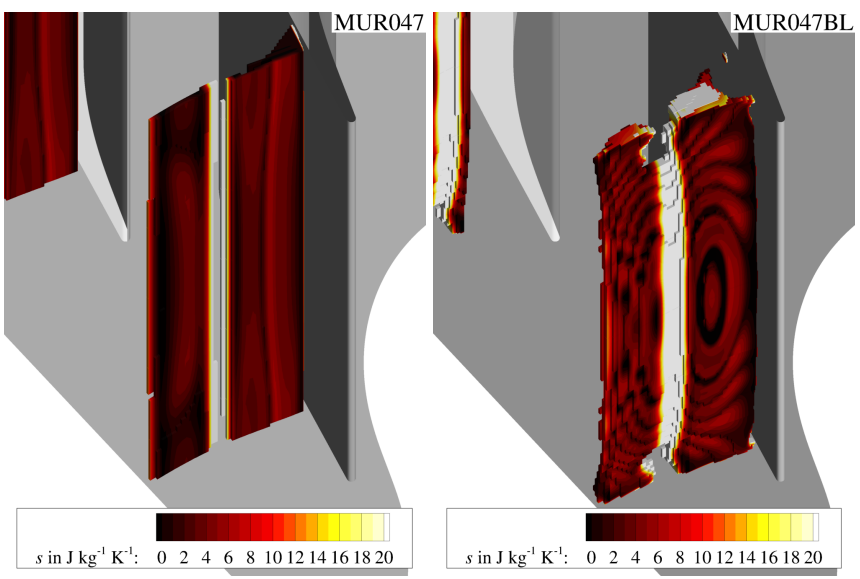

Figure 8 Shock comparison with specific entropy contour 
What is the cause of this structure? Figure 9 shows the vorticity distribution of streamwise vorticity in a series of planes roughly parallel to the shock surface. Beside zones of high streamwise vorticity, that indicated the presence of vortex cores, regions of considerably smaller, yet non-zero streamwise vorticity are visible. These regions are a result of vorticity diffusing from the vortex cores to their surroundings, and by means of vorticity diffusion they grow in cross-sectional area, until vortex sheets form in span-wise-stream-wise direction as indicated in Fig. 9 a). As the vortex sheets encounter the shock-which is perpendicular to the orientation of the vorticity vector-they incur the formation of wave-like instabilities and spots of locally elevated streamwise vorticity, leading to the characteristic "ripple"-pattern mentioned previously (see Fig. 9 d). This pattern gains in intensity while passing the shock and begins to vanish shortly after.

An attempt at explaining the underlying mechanism, which leads to the formation of this concentric pattern, is illustrated in Fig. 10: the diagram shows different stages of a vortex sheet between flow regions of positive and negative streamwise vorticity during the formation process. The stages of the vortex sheet are denoted by the letters a)-e) and give a rough representation of the hub-near vortex sheet in Fig. 9 a)-e). For a), it is assumed that the vortex sheet experiences an initial disturbance. In the case considered in this paper, the initial disturbance is likely imposed by the strong vortex cores already present (see Fig. 9 a). As can be seen in the upper fifth of Fig. 10, the vorticity distribution induces a flow with the induced velocity $c_{\text {ind }}$ along the vortex sheet. The three purple arrows represent the induced velocities at characteristic points of the vortex sheet, indicating that the vortex sheet experiences a movement towards the left near the topmost purple arrow, an upward movement for the middle purple arrow and a movement towards the right for the bottommost purple arrow. The strong streamwise velocity gradients and the resulting vortex deformation across the shock amplifies this effect, leading to a growth of the instability as depicted by the dashed lines b)-e) and hinted by the curved arrows. We suppose that this eventually leads to the "ripple"-pattern observed in this paper.

\section{CONCLUSIONS}

In this paper, we have considered the interaction between the secondary flow system and a normal compression shock in the transonic turbine cascade VKI LS89. In order to isolate the impact of secondary flow, we have computed two test cases numerically: the reference test case features no inlet boundary layer and inviscid end walls and, hence, no secondary-flow vortices. The other test case features rather thick inlet boundary layers, viscous end-wall treatment and, therefore, develops considerable secondary flow structures.

We have introduced an approach to separate losses in a transonic cascade, based on the loss-generating flow features, which can be further extended and adapted to different cases. The analysis reveals the wakes to be the dominant loss-generating mechanism. While the introduction of secondary flow reduces
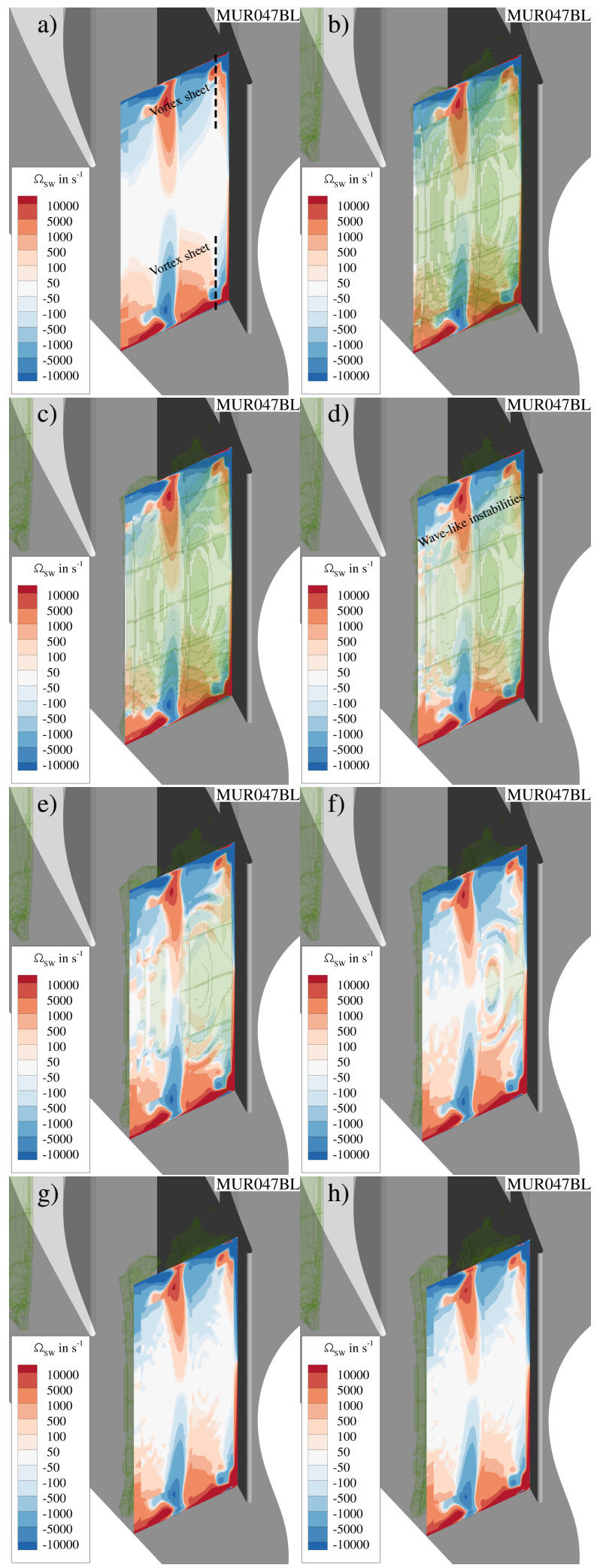

Figure 9 Interaction of secondary flow with compression shock (depicted in green) at various planes $(a-h)$ traversing the shock surface in streamwise direction 


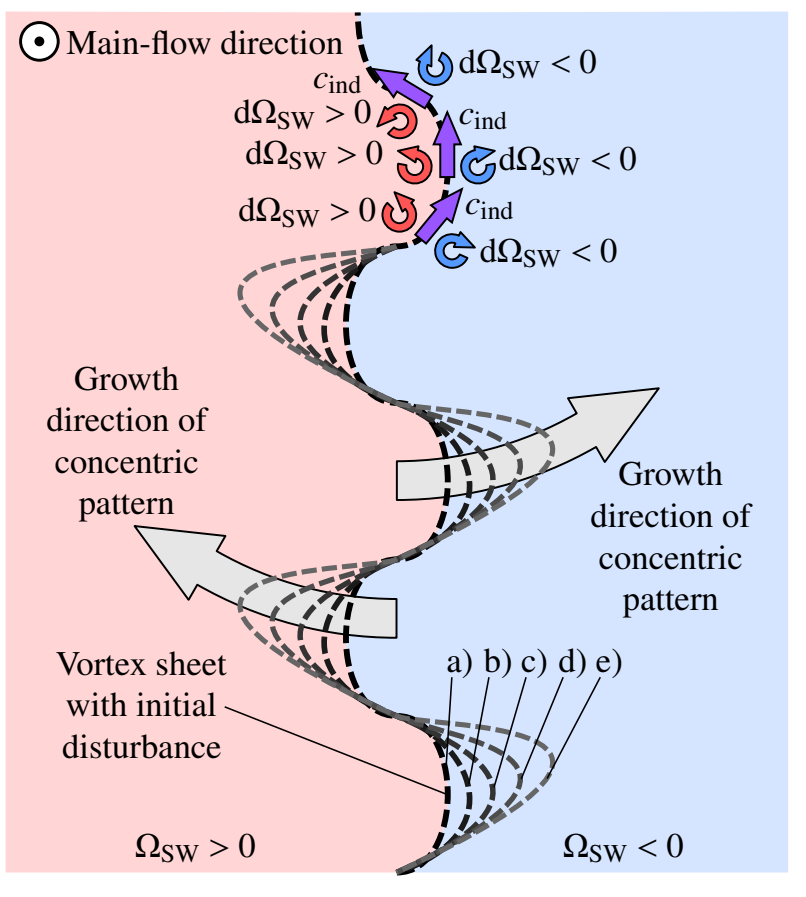

\section{Figure 10 Initial disturbance in a vortex sheet (a) grows to form a concentric pattern (b-e)}

wake losses, the end-wall boundary layer losses and secondaryflow losses increase significantly, leading to an overall rise in entropy.

We have shown that the sudden deceleration caused by the compression shock triggers an instability in weaker regions of the vortex system. As a consequence, a pattern of concentric rings of increased vorticity emerges in the vicinity of the shock. These concentric vorticity regions, in turn, deform the shock front slightly, causing the shock surface to form the same concentric pattern of "ripples". Due to the resulting uneven shock-intensity distribution, the same pattern appears in the entropy distribution just downstream of the shock.

Based on the similarity between the mechanisms that produce turbulence and dissipate kinetic energy, we expect similar instabilities to have a marked impact on turbulence behaviour. Due to limitations and uncertainties of the RANS approach, however, scale-resolving simulations are required to investigate this aspect further.

\section{ACKNOWLEDGEMENTS}

We gratefully acknowledge the substantial contribution of the DLR Institute of Propulsion Technology and MTU Aero Engines AG for providing TRACE. We would like to thank $\mathrm{Mr}$ Allen George, who performed the geometry optimisation for the VKI LS89 profile and generated the computational meshes. We would also like to thank Professor Arul Prakash for initiating $\mathrm{Mr}$ George's research internship at the Institute of Turbomachinery and Fluid Dynamics (TFD) of the Leibniz University Hannover.

\section{NOMENCLATURE Latin Symbols}

$\begin{array}{ll}a & \text { speed of sound } \\ c_{p} & \text { specific heat capacity at constant pressure } \\ c, \mathbf{c} & \text { velocity, velocity vector } \\ e & \text { relative error } \\ \mho_{\mathrm{S}} & \text { factor of safety } \\ F_{y} & \text { pitch-wise blade force, i.e., lift } \\ h & \text { channel height } \\ \ell, \ell_{x} & \text { chord length, axial chord length } \\ \dot{m} & \text { mass-flow rate } \\ \mathrm{Ma} & \text { Mach number } \\ N & \text { node count } \\ p & \text { pressure } \\ R & \text { specific gas constant } \\ \mathfrak{R} & \text { refinement ratio } \\ S, S & \text { specific entropy, entropy } \\ T & \text { temperature } \\ \text { Tu, } l_{\text {turb }} & \text { turbulence intensity, turbulent length scale } \\ V, V & \text { volume, control volume } \\ w & \text { weighting function } \\ x, y, z & \text { axial, pitch-wise, span-wise coordinate } \\ y^{+} & \text {dimensionless wall distance }\end{array}$

\section{Greek Symbols}

$\alpha \quad$ pitch-wise angle

$\delta \quad$ turning angle

$\epsilon \quad$ distance

$\zeta \quad$ total pressure loss coefficient

$\kappa \quad$ heat capacity ratio

$\rho$ density

$\varrho \quad$ observed order of accuracy

$\sigma \quad$ shock criterion

$\psi \quad$ Boolean function

$\Omega, \Omega \quad$ vorticity, vorticity vector

\section{Subscripts}

$\begin{array}{ll}\text { CW, SW } & \text { crosswise, streamwise } \\ \text { in, out } & \text { inlet, outlet } \\ \text { ind } & \text { induced } \\ \text { is } & \text { isentropic } \\ \text { ref } & \text { reference value } \\ \text { tot } & \text { total quantity }\end{array}$

Acronyms

CS compression shock

EW end wall

EWBL end-wall boundary layer

GCI grid-convergence index

EERE estimated extrapolated relative error

PBL profile boundary layer

RANS Reynolds-averaged Navier-Stokes

SF secondary flow

TE trailing edge

W wake 


\section{APPENDIX A: DEFINITIONS}

$$
\begin{aligned}
\psi_{\sigma}(\sigma) & \equiv \begin{cases}1 & \text { if } \quad \sigma \geq 1 \\
0 & \text { else. }\end{cases} \\
\psi_{z}(\boldsymbol{\Omega}) & \equiv \begin{cases}1 & \text { if } \quad \Omega_{z}^{2}>\left(\Omega_{x}^{2}+\Omega_{y}^{2}\right) \\
0 & \text { else. }\end{cases} \\
\psi_{\mathrm{P}}\left(x_{\mathrm{TE}}, \epsilon\right) & \equiv \begin{cases}1 & \text { if } \quad x \leq x_{\mathrm{TE}}+\epsilon \text { with } \epsilon=2 \mathrm{~mm} \\
0 & \text { else. }\end{cases} \\
\psi_{\mathrm{EW}}(y) & \equiv \begin{cases}1 & \text { if } \quad y / h \leq 0.15 \\
1 & \text { if } \quad y / h \geq 0.85 \\
0 & \text { else. }\end{cases}
\end{aligned}
$$

\section{APPENDIX B: BLENDING FUNCTIONS}

$$
\begin{aligned}
s_{\mathrm{SF}} & \equiv w_{\mathrm{SW}} s \\
s_{\mathrm{CS}} & \equiv \psi_{\sigma} w_{\mathrm{CW}} s \\
s_{\mathrm{PBL}} & \equiv\left(1-\psi_{\sigma}\right) \psi_{z} \psi_{\mathrm{P}} w_{\mathrm{CW}} s \\
s_{\mathrm{EWBL}} & \equiv\left(1-\psi_{\sigma}\right)\left(1-\psi_{z}\right)\left(1-\psi_{\mathrm{P}}\right) \psi_{\mathrm{EW}} w_{\mathrm{CW}} s \\
s_{\mathrm{W}} & \equiv\left(1-\psi_{\sigma}\right)\left(1-\psi_{\mathrm{EW}}\right) w_{\mathrm{CW}} s
\end{aligned}
$$

\section{References}

[1] Puterbaugh, S. L. and Brendel, M. "Tip Clearance Flow - Shock Interaction in a Transonic Compressor Rotor". In: Journal of Propulsion and Power 13.1 (1997). DoI: 10.2514/2.5146.

[2] Thomer, O., Schröder, W., and Krause, E. "Normal and Oblique Shock-Vortex Interaction". In: Proceedings of International Conference RDAMM2001. Vol. 6. 2. 2001.

[3] Zudov, V. N. "Interaction of a Streamwise Vortex with the Normal Shock". In: Journal of Applied Mechanics and Technical Physics 52.5 (2011), pp. 734-743. Dor: 10.1134/SO021894411050075.

[4] Graham, C. G. and Kost, F. H. "Shock Boundary Layer Interaction on High Turning Transonic Turbine Cascades". In: Proc. ASME. 79672. Vol. 1A. Paper No. 79GT-37. 1979. DoI: 10.1115/79-GT- 37.

[5] Perdichizzi, A. "Mach Number Effects on Secondary Flow Development Downstream of a Turbine Cascade". In: Proc. ASME. 79139. Vol. 1. Paper No. 89-GT-67. 1989. Dor: $10.1115 / 89-\mathrm{GT}-67$.

[6] Arts, T., Rouvroit, M. L. de, and Rutherford, A. W. AeroThermal Investigation of a Highly Loaded Transonic Linear Turbine Guide Vane Cascade. A test case for inviscid and viscous flow computations. Technical Note 174. von Karman Institute for Fluid Dynamics, 1990.

[7] Detemple-Laake, E. "Detailed Measurements of the Flow Field in a Transonic Turbine Cascade". In: vol. 1. Paper No. 91-GT-029. 1991. DoI: 10. 1115/91-GT-029.
[8] Moustapha, S. H., Carscallen, W. E., and McGeachy, J. D. "Aerodynamic Performance of a Transonic Low Aspect Ratio Turbine Nozzle”. In: J. Turbomach. 115.3 (1993), pp. 400-408. Dor: 10.1115/1.2929267.

[9] Menter, F. R. "Two-Equation Eddy-Viscosity Turbulence Models for Engineering Applications". In: AIAA Journal 32.8 (1994), pp. 1598-1605. Dor: 10.2514/3. 12149.

[10] Menter, F., Kuntz, M., and Langtry, R. B. "Ten Years of Industrial Experience with the SST Turbulence Model". In: Turbulence, Heat and Mass Transfer 4 (2003), pp. 625-632. DoI: 10.1.1.460.2814.

[11] Kato, M. and Launder, B. E. "The Modelling of Turbulent Flow Around Stationary and Vibrating Square Cylinders". In: 9th Symposium on Turbulent Shear Flows. 1993, pp. 10.4.1-10.4.6.

[12] Wheeler, A. P. S., Sandberg, R. D., Sandham, N. D., Pichler, R., Michelassi, V., and Laskokski, G. "Direct Numerical Simulations of a High-Pressure Turbine Vane". In: $J$. Turbomach. 138.7 (2016). DoI: 10.1115/1. 4032435.

[13] Roache, P. J. "Perspective: A Method for Uniform Reporting of Grid Refinement Studies". In: J. Fluids Eng. 116.3 (1994), pp. 405-413. Dor: 10.1115/1.2910291.

[14] ASME V\&V Committee. Standard for Verification and Validation in Computational Fluid Dynamics and Heat Transfer. The American Society of Mechanical Engineers, 2009.

[15] Oettinger, M., Mimic, D., Henke, M., Schmunk, O., and Seume, J. R. "Loss Assessment of the Axial-Gap Size Effect in a Low-Pressure Turbine". In: Proceedings of Global Power and Propulsion Society Beijing Conference 2019. Paper No. GPPS-BJ-2019-0207. 2019.

[16] Lovely, D. and Haimes, R. "Shock Detection from Computational Fluid Dynamics Results ". In: 14th Computational Fluid Dynamics Conference. Paper No. AIAA 99-3285. 1999. 